\title{
Treatment of early bulky cervical cancer with neoadjuvant paclitaxel, carboplatin and cisplatin prior to laparoscopical radical hysterectomy and pelvic lymphadenectomy
}

\author{
YI SHEN, LU YANG and ZEHUA WANG \\ Department of Gynaecology and Obstetrics, Union Hospital, Tongji Medical College, \\ Huazhong University of Science and Technology, Wuhan, Hubei, P.R. China
}

Received June 17, 2011; Accepted September 29, 2011

DOI: $10.3892 / \mathrm{ol} .2011 .529$

\begin{abstract}
The objective of this study was to evaluate the efficacy and safety of neoadjuvant chemotherapy (NAC) with paclitaxel combined with carboplatin and cisplatin prior to laparoscopic radical hysterectomy and pelvic lymphadenectomy. A total of 19 patients who had bulky cervical cancer of International Federation of Gynecology and Obstetrics (FIGO) Stage Ib2 to IIb2 (lesion diameter $>4 \mathrm{~cm}$ ) received NAC with paclitaxel combined with carboplatin and cisplatin prior to laparoscopic radical hysterectomy and pelvic lymphadenectomy. The tumor diameter, the response rate, the resection rate and the lymph node metastasis rate after chemotherapy, as well as the toxicity were evaluated. All patients successfully received NAC, with the exception of 1 patient with FIGO Stage Ib2 and $7 \mathrm{~cm}$ tumor diameter, who rejected complete therapy. The tumor diameter was reduced from $4.93 \pm 0.81 \mathrm{~cm}$ before NAC to $2.57 \pm 1.90 \mathrm{~cm}$ after treatment $(\mathrm{P}<0.01)$, and the overall response rate $[$ complete remission $(\mathrm{CR})+$ partial remission (PR)] was $78.9 \%(15 / 19)$. Two patients received radiotherapy after $\mathrm{NAC}, 1$ patient was found to have cystic metastasis during the surgery and the operative rate was $83.3 \%$ (15/18). Pelvic lymph node metastasis was found in 2 of the 18 patients $(11.1 \%)$ without parametrial and vaginal margin involvement. Hematological toxicity was the most common side effect and the 3-4 toxicity level was only $11.1 \%(2 / 18)$. In conclusion, NAC with paclitaxel, combined with carboplatin and cisplatin, followed by laparoscopic radical hysterectomy and pelvic lymphadenectomy was found to be effective and safe. Thus, NAC is the ideal treatment for patients with early bulky cervical cancer.
\end{abstract}

\section{Introduction}

Cervical cancer is one of the common malignant tumors in women, with the traditional treatment of early cervical cancer

Correspondence to: Dr Zehua Wang, No. 1277, Jiefang Road, Wuhan, Hubei, P.R. China

E-mail: zehuawang@163.com

Key words: neoadjuvant chemotherapy, cervical cancer, paclitaxel being radical surgery or radiation therapy. However, there is some controversy regarding the treatment for bulky cervical cancer (lesion diameter $>4 \mathrm{~cm}$ ) or locally advanced cervical cancer. This is due to the limitation that the bulky mass exceeds the scope of surgical resection, and with the gradual growth of tumor diameter, the radiation dose required far exceeds the tolerance of the surrounding normal tissues. Therefore, surgery and radiation therapy have not proven successful with regards to the treatment of bulky cervical cancer (1). Advances in the research and development of the disease have resulted in observations that cervical cancer is a tumor that is sensitive to chemotherapy (2). New treatment strategies, such as neoadjuvant chemotherapy (NAC), have been established, for example, chemotherapy administered prior to the treatment of cancer can be differentiated from the second-line treatment following surgery (3).

Since Friedlander reported the application of NAC on cervical cancer in 1983, research findings from a large number of phase II and III clinical trials have been reported. However, as the studies consisted of different clinical phase cases and various chemotherapy regimens, the response rate of NAC has been questioned. In addition, there is no standardized route of administration or course of treatment for NAC $(4,5)$. Paclitaxel is now considered a new type of broad-spectrum and highly efficient anticancer drug, and the efficacy of this agent on a variety of solid tumors has been noted. For example, one study has confirmed that paclitaxel kills the human cervical cancer cell $\mathrm{SiHa}$ in vitro (6). In the current study, paclitaxel combined with carboplatin and cisplatin therapy was administered to patients between January 2009 and June 2010, who had bulky cervical cancer (FIGO stage Ib2 to IIb2) and who had received NAC prior to laparoscopic radical hysterectomy and pelvic lymphadenectomy, in order to explore the response rate and feasibility of this method on the treatment of bulky cervical cancer.

\section{Materials and methods}

Case selection. A total of 19 patients (average age 42.5, range 29-62) with bulky cervical cancer who were treated with NAC (paclitaxel combined with carboplatin systemic chemotherapy and cervical local injection of cisplatin) between 
January 2009 and June 2010 in Wuhan Union Hospital (China) were enrolled in the current study. After 3 courses of treatment, patients were treated with laparoscopic radical hysterectomy and pelvic lymphadenectomy. Initial treatment and diagnoses of the patients were confirmed by cervical biopsy prior to surgery. The clinical stage was identified in accordance with the standards issued by the International Federation of Gynecology and Obstetrics (FIGO) in 2000, and cervical local lesion size was measured by magnetic resonance imaging (MRI).

Treatment methods. Patients studied were initially treated with NAC drugs as follows: cisplatin $(20 \mathrm{mg}$, cervical local injection) was applied for chemotherapy $\mathrm{d} 1$ and $\mathrm{d} 4$, paclitaxel (trade name: liposome, $30 \mathrm{mg} /$ bottle, H20030357, batch no.: 070606, Nanjing Sike Pharmaceutical Co., Ltd.; Nanjing, China) $\left(150 \mathrm{mg} / \mathrm{m}^{2}\right.$ intravenous infusion) for $\mathrm{d} 2$ and carboplatin (i.v., AUC=5) for d3. Each course of medical treatment was admini-stered every 28 days. The side effects were closely observed during chemotherapy and were evaluated according to the standard of chemotherapy toxic response issued by the World Health Organization (WHO). Gynecological examination was carried out prior to each chemotherapy course in order to initially assess the lesion development and whether there was progression of the clinical stage. If the lesion progressed or parametrial invasion was aggravated, the NAC was replaced by radiotherapy. Re-examination of MRI was performed prior to therapy in order to evaluate the efficacy of chemotherapy.

Following administration of chemotherapy, laparoscopic radical hysterectomy and pelvic lymphadenectomy were performed. The specific surgical procedures are available in our previous reports (7). For the patients with lymph node metastasis, parametrial involvement or positive vaginal margin after surgery, adjuvant radiotherapy was carried out.

Evaluation of chemotherapy. The efficacy of chemotherapy was evaluated according to the WHO evaluation criteria: 1, complete remission (CR): tumor disappeared completely with no lymph node metastasis; 2, partial remission (PR): at least $50 \%$ tumor shrinkage; 3 , stable disease (SD): tumors shrank by $<50 \%$ or enlarged by $<25 \%$; 4 , progression disease (PD): tumors enlarged by $>25 \%$ or there was a new lesion. Effective chemotherapy refers to patients with CR or PR, ineffective chemotherapy refers to patients with SD or PD.

Follow-up. Out-patient follow-up was conducted for all 19 patients, once every three months after the first year of therapy. Follow-up examination consisted of gynecological examination, vaginal stump brushing cytology examination, pelvic B-us, B-us examination of kidneys, ureters and bladder, and chest examination.

\section{Results}

The average age of the 19 patients was 42.5 (29-62 years old), and all the individuals had completed at least one course of chemotherapy and undergone the corresponding evaluation (Table I). The average diameter of the bulky tumors was $4.93 \pm 0.81 \mathrm{~cm}(4.0-7.0 \mathrm{~cm})$.
Table I. Clinical data of the cases $(n=19)$.

Clinical features

Age (years, range)

$42.5 \pm 8.2(29-62)$

Clinical stage (FIGO)

$\mathrm{Ib} 2$

9

IIa2

IIb2

Pathology

Squamous carcinoma $\quad 14$

Adenocarcinoma 3

Adenosquamous carcinoma

3

FIGO, International Federation of Gynecology and Obstetrics.

Reduction of tumor size and response to NAC. All 19 patients had undergone MRI examination and measurement of the cervical lesion size prior to NAC and following the chemotherapy (Table II and Fig. 1). The diameter of the tumor after chemotherapy was markely reduced to $2.57 \pm 1.90 \mathrm{~cm}(\mathrm{P}<0.01$, vs. the diameter prior to chemotherapy). As confirmed by pathological diagnosis following chemotherapy, there were 4 CR cases $(21.1 \%), 11$ PR cases $(57.9 \%)$ and 4 SD cases (21.1\%), while no PD was observed. The chemotherapy response rate (i.e., CR + PR) was 78.9\% (15/19) (Table III). Of the 19 cases, 1 ( $\mathrm{Ib} 2)$ case reached the maximum lesion diameter of $7.0 \mathrm{~cm}$, with no obvious shrinkage of the lesion after a single course of chemotherapy. Therefore, the patient rejected chemotherapy using radiation therapy. For the remaining 18 patients, no significant remission was observed for 1 IIb2 and $1 \mathrm{Ib} 2$ cases following chemotherapy, therefore radiation therapy was proposed. The remaining case with SD strongly requested surgery; however, this 1 IIa2 case was found to have been infiltrated by the tumor at the bladder serosa during the surgery, and the procedure was terminated. Thus, 15 patients completed the surgery with the surgical resection rate at $83.3 \%$. Of these 15 patients, only two cases, $1 \mathrm{Ib} 2 \mathrm{SD}$ case, who requested surgery, and 1 IIa2 PR case, with lymph node metastasis after surgery were observed. The lymph node metastasis rate was $13.3 \%$, and no parametrial involvement and positive vaginal surgical margin cases were observed.

Side effects of chemotherapy. The side effects caused by paclitaxel combined with carboplatin systemic chemotherapy and local injection of cisplatin were evaluated in accordance with the standards of toxic response issued by WHO. Of the 19 patients studied, 1 discontinued treatment following a single course of chemotherapy; therefore the chemotherapy completion rate was $18 / 19(94.7 \%)$. The most common toxicity was hematological toxicity, 11 cases $(61.1 \%)$ presented with decreased white blood cell and granulocyte cell counts, of which 2 cases $(11.1 \%)$ had a 3-4 level white blood cell and granulocyte decrease. The incidence of thrombocytopenia was 3/18 (16.7\%), and no 3-4 level of thrombocytopenia was observed. Minor gastrointestinal reactions were evident; 3 cases $(16.7 \%)$ presented with mild nausea and vomiting. No peripheral nervous system side effects occurred. Of the 18 cases, 1 (5.6\%) presented with liver damage, 
Table II. Tumor size before and after NAC.

\begin{tabular}{|c|c|c|c|}
\hline Stage & $\begin{array}{c}\text { Before NAC } \\
\text { tumor size } \\
\text { (height } \mathrm{x} \text { length } \mathrm{x} \text { width) } \mathrm{cm}\end{array}$ & $\begin{array}{c}\text { After NAC } \\
\text { tumor size } \\
\text { (height } \mathrm{x} \text { length } \mathrm{x} \text { width) } \mathrm{cm}\end{array}$ & Note \\
\hline \multirow[t]{9}{*}{$\mathrm{Ib} 2$} & $4.5 \times 4.0$ & $2.0 \times 2.0$ & \\
\hline & $4.2 \times 3.5 \times 2.7$ & No obvious mass & \\
\hline & $4.2 \times 4.5$ & $1.0 \times 1.5$ & \\
\hline & $5.4 \times 4.3 \times 3.0$ & $5.0 \times 4.5 \times 3.0$ & \\
\hline & $5.5 \times 3.9$ & No obvious mass & \\
\hline & $4.1 \times 3.6 \times 4.0$ & $1.0 \times 1.5$ & \\
\hline & $7.0 \times 4.5$ & $6.5 \times 5.0$ & $\begin{array}{l}\text { Rejected after one } \\
\text { course of chemotherapy }\end{array}$ \\
\hline & $4.0 \times 3.0 \times 5.3$ & $2.0 \times 1.5$ & \\
\hline & $6.0 \times 5.0$ & $4.0 \times 5.5$ & \\
\hline \multirow[t]{9}{*}{ IIa2 } & $4.1 \times 3.9 \times 4.2$ & $3.0 \times 2.5$ & \\
\hline & $6.0 \times 4.9 \times 5.0$ & $2.5 \times 3.0 \times 2.2$ & \\
\hline & $4.5 \times 2.0 \times 5.0$ & $3.0 \times 2.0 \times 2.5$ & \\
\hline & $5.0 \times 3.0$ & $2.9 \times 1.3$ & \\
\hline & $4.0 \times 4.1 \times 3.5$ & $2.2 \times 3.5 \times 2.2$ & \\
\hline & $3.0 \times 3.5 \times 4.0$ & No obvious mass & \\
\hline & $5.1 \times 3.9 \times 4.0$ & $2.5 \times 3.0 \times 2.0$ & \\
\hline & $3.8 \times 3.4 \times 5.2$ & No obvious mass & \\
\hline & $3.5 \times 4.0 \times 4.1$ & $1.5 \times 2.0 \times 1.5$ & \\
\hline $\mathrm{IIb} 2$ & $4.5 \times 3.5$ & $4.5 \times 4.0$ & \\
\hline
\end{tabular}

NAC, neoadjuvant chemotherapy.

A

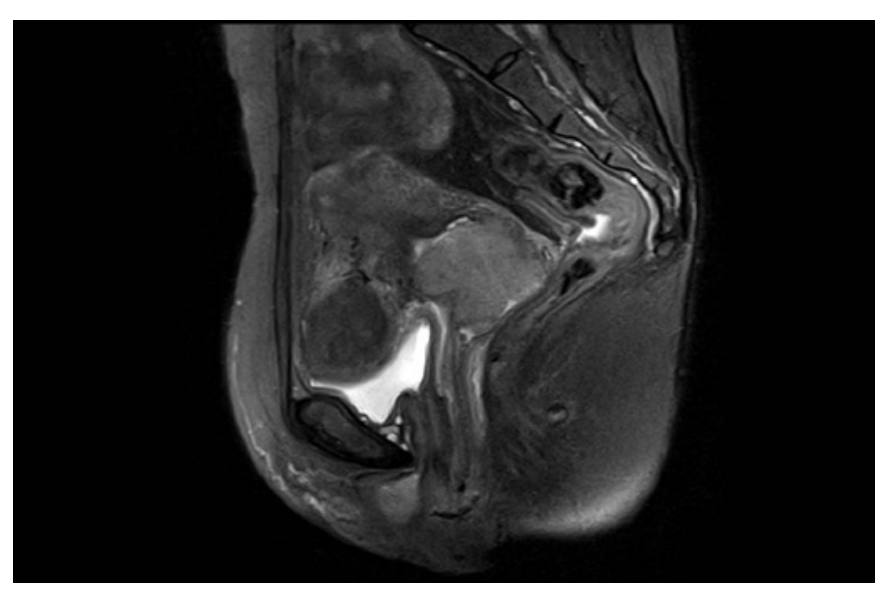

B

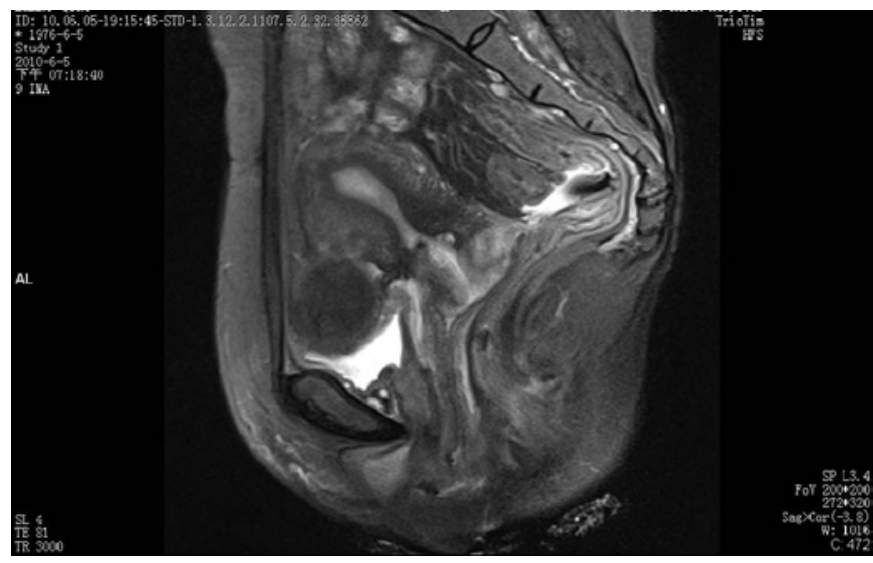

Figure 1. MRI results for cervical cancer lesions before and after neoadjuvant chemotherapy. (A) MRI prior to neoadjuvant chemotherapy. A 3.8x3.4x5.2 cmsized mass in the cervix was observed, which involved the vaginae fornix. (B) MRI following 3 courses of chemotherapy; the lesion disappeared, no obvious mass was observed and the vaginae fornix involvement was ameliorated. MRI, magnetic resonance imaging.

chemotherapy was continued after liver protection and enzyme reduction treatment, and there was no renal dysfunction. No drug allergy was observed in any of the patients.

Follow-up after surgery. With the exception of 1 case who discontinued NAC, all patients were followed up until the last day, July 2010. No cases of recurrence or metastasis after surgery have been observed as yet.

\section{Discussion}

The efficacy of NAC on the treatment of bulky or locally advanced cervical cancer remains to be determined. NAC is potentially capable of reducing localized lesions, improving parametrial involvement and increasing the surgical resection rate (8). NAC prior to radiotherapy or surgery-induced blood rheology variation in the local tumor is beneficial for the 
Table III. Analysis of neoadjuvant chemotherapy response rate.

\begin{tabular}{lccccc}
\hline \multirow{2}{*}{ Chemotherapy response } & \multicolumn{3}{c}{ Clinical stage (case) } & Total & Ratio (cases/total cases) \% \\
\cline { 2 - 4 } & Ib2 & IIa2 & IIb2 & & 21.1 \\
\hline CR & 2 & 2 & 0 & 4 & 57.9 \\
PR & 4 & 7 & 0 & 11 & 21.1 \\
SD & 3 & 0 & 1 & 4 & 0 \\
PD & 0 & 0 & 0 & 0 & 19 \\
Total & 9 & 9 & 1 & & \\
\hline
\end{tabular}

control or elimination of pelvic micrometastases as it decreases the lymph node metastasis rate, thereby reducing the radiotherapy rate, decreasing the risk of recurrence and improving quality of life following surgery. Recent meta-analyses have confirmed this hypothesis (3). However, on the basis of a randomized, controlled study of stage Ib2-IIb cervical cancer, Katsumata et al found that there was no significant difference between a NAC-combined surgery group and the surgery alone group in terms of resection and survival rate (9). This discrepancy is attributed to the differences of chemotherapy regimens, courses and case selections. Since platinum drugs, particularly cisplatin, are sensitizers of radiation therapy, early research on NAC has mainly focused on cisplatin-based chemotherapy, and numerous investigators have confirmed that the response rate of cisplatin-combined duplex treatment is superior to that of single treatment with cisplatin (10). The application of paclitaxel provides a new option for NAC chemotherapy. One randomized controlled study in Italy has shown that the chemotherapy remission rate in ifosfamide and cisplatin combined with the paclitaxel (TIP) group was significantly increased compared with that in the paclitaxel-negative group (IP) (48 vs. $23 \%$ ), and that the survival time for patients who achieved CR and PR was longer than that of patients with a poor response to chemotherapy (11). Moore et al confirmed that a chemotherapy response rate of $36 \%$ can be achieved in paclitaxel combined with cisplatin NAC (12). Thus, paclitaxel-based NAC specimens have attracted extensive attention.

An advantage of NAC involves its ability to reduce tumor size, improving the resection rate and controlling the lymph node metastasis rate. To accurately evaluate changes in the tumor volume, the patients included in this study were examined by MRI prior to and following chemotherapy in order to ensure the accuracy of the assessment. We confirmed that the lesion size in all cases after NAC was reduced to varying degrees; the tumor diameter prior to chemotherapy was reduced from $4.93 \pm 0.81 \mathrm{~cm}$ to $2.57 \pm 1.90 \mathrm{~cm}$ after chemotherapy $(\mathrm{P}<0.01)$. Even the 7 - $\mathrm{cm}$ cervical lesion of the Ib2 case was reduced to $6.5 \mathrm{~cm}$ after only a single course of chemotherapy. However, the patient eventually discontinued therapy due to financial reasons. Findings of various reports have shown that the response rate of paclitaxel-based NAC on the treatment of bulky and locally advanced cervical cancer was $36-95 \%$, and the CR rate was 9-39\% (12-16). However, no cervical local injection method was observed in any of the programs. In the present study, paclitaxel-based systemic and local chemotherapy were studied. The effective rate reached
$78.9 \%$ and the CR rate reached $21.1 \%$, which was second only to the $39 \%$ CR rate reported by Park et al (13). However, the course applied by these authors was 10 days/course, which was shorter and had greater side effects (13). Cervical local injection was applied in this study, which reduced the toxicity of drugs and, at the same time, increased the rate of the direct effect on the lesions. Of the 18 cases, 4 were pathologically confirmed as the lesion having completely disappeared, demonstrating that the method was safe and therapeutically effective. In addition, 15 cases completed the surgery with a surgical resection rate of $83.3 \%$, which is in line with the removal rate of other countries of $73-100 \%$ (17). Two cases presented with lymph node metastasis following surgery with a metastasis rate of $13.3 \%$, which is slightly lower than the transfer rate of $22-25 \%$ noted in other studies (18). Therefore, NAC (i.e., paclitaxel combined with carboplatin and cisplatin systemic and local chemotherapy) on the treatment of bulky cervical cancer is effective. However, a limited number of patients was included in the present study. Therefore, a larger cohort of patients is required in future studies to confirm our results.

The toxicity of NAC is one of the main elements of focus. The most common side effects in paclitaxel combined with platinum drugs are blood system and renal toxicity. However, the toxic response to level 3-4 is relatively small, and it is capable of being mitigated through symptomatic treatment. In this study, $61.1 \%$ of the patients presented with bone marrow suppression, mainly white blood cell and granulocyte decrease, but only 2 cases were level 3-4, which were ameliorated after granulocyte colony-stimulating factor treatment, and no serious infection was observed. On the basis of reducing allergic reaction and maintaining the response rate, paclitaxel liposome was applied. No cases of allergic reaction were observed in the study. With the exception of systemic treatment, there was local cervical injection of cisplatin. As for the response rate, this method of treatment is capable of significantly reducing the lesion size since cisplatin is unable to directly infiltrate into the blood; the side effects caused by cisplatin, such as bone marrow suppression and renal toxicity, were lighter than with systemic chemotherapy. Therefore, even if combined with local treatment, no serious liver or kidney damage was observed, with the exception of 1 case with mild liver dysfunction. Therefore, the chemotherapy regimen applied in this study is safe and feasible.

In conclusion, NAC with paclitaxel, combined with carboplatin and cisplatin, for the treatment of bulky cervical cancer is capable of reducing tumor volume and improving the 
operative rate effectively with low toxicity. It is therefore capable of improving the survival rate and reducing the recurrence of cancer. However, NAC should be investigated in a larger cohort of patients in randomized prospective studies in order to confirm results obtained in the present study.

\section{References}

1. Eddy GL, Bundy BN, Creasman WT, et al: Treatment of ('bulky') stage IB cervical cancer with or without neoadjuvant vincristine and cisplatin prior to radical hysterectomy and pelvic/para-aortic lymphadenectomy: a phase III trial of the gynecologic oncology group. Gynecol Oncol 106: 362-369, 2007.

2. Benedetti Panici P, Greggi S, Scambia G, et al: Long-term survival following neoadjuvant chemotherapy and radical surgery in locally advanced cervical cancer. Eur J Cancer 34: 341-346, 1998.

3. Choi CH, Kim T, Lee J, et al: Phase II study of neoadjuvant chemotherapy with mitomycin-c, vincristine and cisplatin (MVC) in patients with stages IB2-IIB cervical cancer. Gynecol Oncol 104: 64-69, 2007.

4. Neoadjuvant Chemotherapy for Locally Advanced Cervical Cancer Meta-analysis Collaboration: Neoadjuvant chemotherapy for locally advanced cervical cancer: a systematic review and meta-analysis of individual patient data from 21 randomised trials. Eur J Cancer 39: 2470-2486, 2003.

5. Bae JH, Lee S, Lee A, et al: Neoadjuvant cisplatin and etoposide followed by radical hysterectomy for stage 1B-2B cervical cancer. Gynecol Oncol 111: 444-448, 2008.

6. Lihong Zhu, Lihui Wei, Xiaoping Li, et al: Killing effect of paclitaxel liposome on cervical carcinoma $\mathrm{SiHa}$ cells in vitro. Bull. Chin Cancer 17: 704-707, 2008.

7. Shen Y and Wang Z: Total laparoscopic radical hysterectomy for treatment of uterine malignant tumors: analysis of short-term therapeutic efficacy. J Huazhong Univ Sci Technol [Med Sci] 30 376-379, 2010.

8. Lai CH, Hsueh S, Chang TC, et al: Prognostic factors in patients with bulky stage IB or IIA cervical carcinoma undergoing neoadjuvant chemotherapy and radical hysterectomy. Gynecol Oncol 64: 456-462, 1997.
9. Katsumata N, Yoshikawa H, Hirakawa T, et al: Phase III randomized trial of neoadjuvant chemotherapy (NAC) followed by radical hysterectomy ( $\mathrm{RH}$ ) versus $\mathrm{RH}$ for bulky stage I/II cervical cancer. ASCO Annu Meet Proc 24: abs. 5013, 2006.

10. Monk B, Huang HQ, Cella D, et al: Quality of life outcomes from a randomized phase III trial of cisplatin with or without topotecan in advanced carcinoma of the cervix: a Gynecologic Oncology Group Study. J Clin Oncol 23: 4617-4625, 2005.

11. Buda A, Fossati R, Colombo N, et al: Randomized trial of neoadjuvant chemotherapy comparing paclitaxel, ifosfamide, and cisplatin with ifosfamide and cisplatin followed by radical surgery in patients with locally advanced squamous cell cervical carcinoma: the SNAP01 (Studio Neo-Adjuvante Portio) Italian Collaborative Study. J Clin Oncol 23: 4137-4145, 2005.

12. Moore DH, Blessing JA, McQuellon MP, et al: Phase III study of cisplatin with or without paclitaxel in stage IVB, recurrent, or persistent squamous cell carcinoma of the cervix: a gynecologic oncology group study. J Clin Oncol 22: 3113-3119, 2004.

13. Park DC, Kim JH, Lew YO, Kim DH and Namkoong SE: Phase II trial of neoadjuvant paclitaxel and cisplatin in uterine cervical cancer. Gynecol Oncol 92: 59-63, 2004.

14. Dueñas-Gonzalez A, López-Graniel C, González-Enciso A, et al: A phase II study of multimodality treatment for locally advanced cervical cancer: neoadjuvant carboplatin and paclitaxel followed by radical hysterectomy and adjuvant cisplatin chemoradiation. Ann Oncol 14: 1278-1284, 2003.

15. D'Agostino G, Distefano M, Greggi S, et al: Neoadjuvant treatment of locally advanced carcinoma of the uterine cervix with epirubicin, paclitaxel and cisplatin. Cancer Chemother Pharmacol 49: 256-260, 2004.

16. Zanetta G, Lissoni A, Pellegrino A, et al: Neoadjuvant chemotherapy with cisplatin, ifosfamide and paclitaxel for locally advanced squamous-cell cervical cancer. Ann Oncol 9: 977-980, 1998.

17. Hwang YY, Moon H, Cho SH, et al: Ten-year survival of patients with locally advanced stage IB-IIB cervical cancer after neoadjuvant chemotherapy and radical hysterectomy. Gynecol Oncol 82: 88-93, 2001.

18. Eddy GL, Manetta A, Alvarez RD, et al: Neoadjuvant chemotherapy with Vincristin and cisplatin followed by radical hysterectomy and pelvic lymphadenectomy for FIGO stage IB bulky cervical cancer: a gynecologic oncology group pilot study. Gynecol Oncol 57: 412-416, 1995. 